\title{
Oxygen evolution reaction: Bifunctional mechanism breaking the linear scaling relationship
}

Cite as: J. Chem. Phys. 152, 104712 (2020); https://doi.org/10.1063/1.5143235

Submitted: 20 December 2019 . Accepted: 01 March 2020 . Published Online: 12 March 2020

(D) Patrick Gono, and (iD) Alfredo Pasquarello

\section{COLLECTIONS}

Paper published as part of the special topic on Oxide Chemistry and CatalysisOXIDE2020
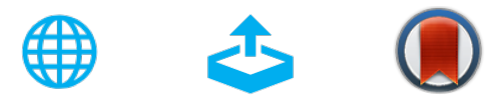

\section{ARTICLES YOU MAY BE INTERESTED IN}

Vibrational properties of $\mathrm{CO}_{2}$ adsorbed on the $\mathrm{Fe}_{3} \mathrm{O}_{4}$ (111) surface: Insights gained from DFT The Journal of Chemical Physics 152, 104702 (2020); https://doi.org/10.1063/1.5136323

The water/ceria(111) interface: Computational overview and new structures

The Journal of Chemical Physics 152, 104709 (2020); https://doi.org/10.1063/1.5142724

Atomic and molecular adsorption on single platinum atom at the graphene edge: A density functional theory study

The Journal of Chemical Physics 152, 104707 (2020); https://doi.org/10.1063/5.0002902

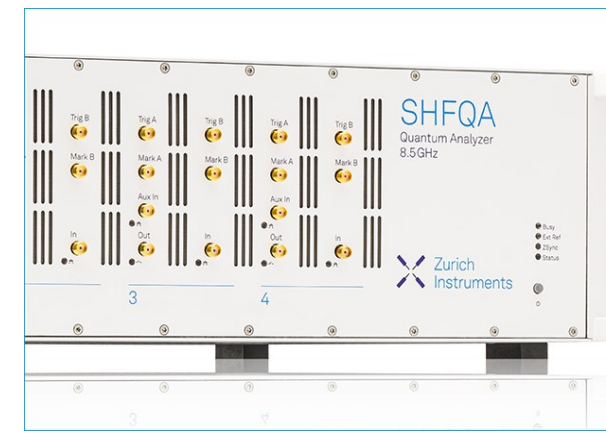

\section{Learn how to perform the readout of up to 64 qubits in parallel With the next generation of quantum analyzers on November 17th}

\section{Register now}

1. Zurich Instruments 


\title{
Oxygen evolution reaction: Bifunctional mechanism breaking the linear scaling relationship
}

\author{
Cite as: J. Chem. Phys. 152, 104712 (2020); doi: 10.1063/1.5143235 \\ Submitted: 20 December 2019 - Accepted: 1 March 2020 • \\ Published Online: 12 March 2020
}

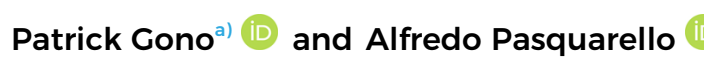

\begin{abstract}
AFFILIATIONS
Chaire de Simulation à l'Echelle Atomique (CSEA), Ecole Polytechnique Fédérale de Lausanne (EPFL), $\mathrm{CH}-1015$ Lausanne, Switzerland
\end{abstract}

Note: This article is part of the JCP Special Topic on Oxide Chemistry and Catalysis.
a) Author to whom correspondence should be addressed: patrick.gono@epf.ch

\begin{abstract}
The bifunctional mechanism for the oxygen evolution reaction (OER) involving two distinct reaction sites is studied through the computational hydrogen electrode method for a set of catalyst materials including rutile $\mathrm{TiO}_{2}(110)$, anatase $\mathrm{TiO}_{2}(101), \mathrm{SnO}_{2}(110)$, $\mathrm{RuO}_{2}(110), \mathrm{IrO}_{2}(110), \mathrm{Ni}_{2} \mathrm{P}(0001)$, and $\mathrm{BiVO}_{4}(001)$. The calculations are performed both at the semilocal level and at the hybrid functional level. Moreover, anodic conditions are modeled and their effect on the OER free energy steps is evaluated. The free energies of the reaction steps indicate that for specific combinations of catalysts, the limitations due to the linear scaling relationship can be overcome, leading to smaller overpotentials for the overall OER. At the same time, a detailed analysis of the results reveals a strong dependence on the adopted functional. For both functionals, it is shown that the energy level of the highest occupied electronic state can serve as a descriptor to guide the search for the optimal catalyst acting as a hydrogen acceptor. These results support the bifunctional mechanism as a means to break the linear scaling relationship and to further reduce the overpotential of the OER.
\end{abstract}

Published under license by AIP Publishing. https://doi.org/10.1063/1.5143235

\section{INTRODUCTION}

Photoelectrochemical water splitting is instrumental in achieving a clean, hydrogen-based fuel economy in the future. ${ }^{1,2}$ Water splitting can be separated into the hydrogen evolution reaction taking place on the cathode and the oxygen evolution reaction (OER) taking place on the anode. A suitable catalyst must, among other things, efficiently catalyze the OER so that no extra potential bias needs to be applied to drive the reaction. Given the plethora of possible anode materials, it is of interest to use computational methods to screen potential water splitting materials for their catalytic properties.

Such screenings have been performed for several classes of materials in the past. ${ }^{3,4}$ A four proton-coupled electron transfer (PCET) mechanism is proposed for the OER in acidic conditions,

$$
\begin{aligned}
\mathrm{H}_{2} \mathrm{O}_{(\ell)} & \longrightarrow \mathrm{OH}_{a}+\mathrm{H}^{+}+\mathrm{e}^{-}, \\
\mathrm{OH}_{a} & \longrightarrow \mathrm{O}_{a}+\mathrm{H}^{+}+\mathrm{e}^{-}, \\
\mathrm{O}_{a}+\mathrm{H}_{2} \mathrm{O}_{(\ell)} & \longrightarrow \mathrm{OOH}_{a}+\mathrm{H}^{+}+\mathrm{e}^{-}, \\
\mathrm{OOH}_{a} & \longrightarrow \mathrm{O}_{2}+\mathrm{H}^{+}+\mathrm{e}^{-},
\end{aligned}
$$

where all the reaction steps occur at a single active site $a$ on the surface of the catalyst. The free energy differences associated with these reactions are then calculated using the computational hydrogen electrode (CHE) method developed by Nørskov et al. ${ }^{6}$

Comprehensive studies of both metallic and non-metallic OER catalysts have uncovered the existence of linear scaling relationships between the free energy steps. ${ }^{3,4,7}$ These linear scaling relationships manifest themselves as a limit on the catalytic efficiency of a wide range of materials. In particular, it was found that the sum of the 
free energies corresponding to the second and third steps of Eq. (1) evaluated at metal and metal-oxide surfaces is equal to $3.2 \mathrm{eV}$. ${ }^{4}$ Since the ideal height of each OER free energy step is $1.23 \mathrm{eV}$, this leads to an overpotential on at least one of these two steps. The existence of these scaling laws is related to the fact that only a single active site is present.

In a recent work by Song et al., a novel catalyst is observed to exhibit activities at variance with the existence of the linear scaling laws. ${ }^{8}$ To explain these observations, a bifunctional OER mechanism has been proposed,

$$
\begin{aligned}
\mathrm{H}_{2} \mathrm{O}_{(\ell)} & \longrightarrow \mathrm{OH}_{a}+\mathrm{H}^{+}+\mathrm{e}^{-}, \\
\mathrm{OH}_{a} & \longrightarrow \mathrm{O}_{a}+\mathrm{H}^{+}+\mathrm{e}^{-}, \\
\mathrm{O}_{a}+\mathrm{H}_{2} \mathrm{O}_{(\ell)} & \longrightarrow \mathrm{H}_{b}+\mathrm{O}_{2}+\mathrm{H}^{+}+\mathrm{e}^{-}, \\
\mathrm{H}_{b} & \longrightarrow \mathrm{H}^{+}+\mathrm{e}^{-},
\end{aligned}
$$

where $a$ and $b$ refer to two different but close active sites. Song et al. suggested a catalyst composed of a $\gamma$ - NiOOH substrate acting as a hydrogen acceptor (site $b$ ) and of $\gamma$-FeOOH nanoparticles hosting the other reaction intermediates (site $a$ ). The inclusion of the hydrogen acceptor effectively sidesteps the problem associated with a single active site and, thus, allows overcoming the limitation associated with the linear scaling of the OER free energy steps.

In this work, we investigate the potential of the bifunctional mechanism in Eq. (2) to reduce the overpotential of the OER for a variety of possible catalysts. We consider the reaction involving both a single and two different sites taking into consideration the following interfaces as model systems: rutile $\mathrm{TiO}_{2}(110)$, anatase $\mathrm{TiO}_{2}(101)$, $\mathrm{SnO}_{2}(110), \mathrm{RuO}_{2}(110), \mathrm{IrO}_{2}(110), \mathrm{Ni}_{2} \mathrm{P}(0001)$, and $\mathrm{BiVO}_{4}(001)$. In addition, we also address to what extent the free energy steps depend on the adopted theoretical framework and, thus, perform calculations at both the semilocal and hybrid-functional levels of theory. Our calculations demonstrate that it is theoretically conceivable that the bifunctional mechanism leads to the breaking of the scaling relationships for a suitable combination of catalysts.

\section{METHODS}

\section{A. Computational details}

All material surfaces are modeled by slabs with rectangular repeat units, separated by $20 \AA$ of vacuum in the direction perpendicular to the interface (see Table I). We determine the lattice parameters by performing a lattice optimization of a bulk supercell, except in the case of $\mathrm{SnO}_{2}$ where we use experimental lattice parameters following Guo et al. ${ }^{9}$ The latter choice is motivated by the fact that small deviations in the lattice parameters strongly affect the calculated energy levels due to the large deformation potential of $\mathrm{SnO}_{2} .{ }^{10}$ The number of layers is chosen so as to achieve bulk-like properties in the center of each slab. All calculations are carried out with the СР2K suite of codes. ${ }^{11}$ The cutoff for the density is set to 600 Ry. The geometry optimizations are carried out without constraints on the positions of the atoms.

The semilocal calculations are carried out with the revised Perdew-Burke-Ernzerhof (RPBE) functional ${ }^{12}$ and yield optimized
TABLE I. From left to right: supercell sizes $L_{x}$ and $L_{y}$ in the $x$ and $y$ directions chosen to lie perpendicular to the surface normal, surface repeat unit, number of layers, and total number of atoms used in the slab model for each material.

\begin{tabular}{lccccc}
\hline \hline & $L_{x}(\AA)$ & $L_{y}(\AA)$ & Periodicity & Layers & Atoms \\
\hline $\mathrm{SnO}_{2}(110)$ & 13.405 & 12.740 & $2 \times 4$ & 5 & 240 \\
$\mathrm{IrO}_{2}(110)$ & 12.870 & 12.709 & $2 \times 4$ & 5 & 240 \\
$\mathrm{RuO}_{2}(110)$ & 12.978 & 12.467 & $2 \times 4$ & 5 & 240 \\
$\mathrm{r}^{-\mathrm{TiO}_{2}(110)}$ & 13.286 & 12.054 & $2 \times 4$ & 5 & 240 \\
$\mathrm{BiVO}_{4}(001)$ & 10.592 & 10.351 & $2 \times 2$ & 3 & 144 \\
$\mathrm{a}^{-\mathrm{TiO}_{2}(101)}$ & 10.522 & 11.431 & $2 \times 3$ & 6 & 180 \\
$\mathrm{Ni}_{2} \mathrm{P}(0001)$ & 11.744 & 10.250 & $2 \times 3$ & 6 & 196 \\
\hline \hline
\end{tabular}

geometries and binding energies of the reaction intermediates. Triple- $\zeta$ MOLOPT basis sets and Goedecker-Teter-Hutter pseudopotentials ${ }^{13}$ are used for all elements. The hybrid functional calculations employ a modified $\mathrm{PBE} 0(\alpha)$ functional ${ }^{14}$ for the semiconductors and a modified $\operatorname{HSE06}(\alpha)$ functional ${ }^{15,16}$ for the metals $\left(\mathrm{IrO}_{2}\right.$, $\mathrm{RuO}_{2}$ ). In the hybrid functional calculations, the structural optimization is carried out consistently with the adopted functional. We use fractions of Fock exchange $\alpha=0.29$ for $\operatorname{PBE} 0(\alpha)$ and $\alpha=0.305$ for $\operatorname{HSE06}(\alpha)$. These values are chosen to ensure that the calculated free energy of the OER $\left(\Delta G_{\mathrm{OER}}\right)$ corresponds to its experimental value (see Sec. II B). Due to the increased computational cost with respect to the semilocal functional, only double- $\zeta$ basis sets are used. Total energy differences in selected cases are found to be modified by less than $0.01 \mathrm{eV}$ due to the smaller basis sets. To speed up the calculations, the auxiliary density matrix method is employed.

\section{B. Computational hydrogen electrode}

In the framework of the CHE method, ${ }^{6}$ the chemical potential of the proton-electron pair is taken to be one half of the chemical potential of the gaseous hydrogen molecule in standard hydrogen electrode conditions. Thus, the free energy corresponding to the reaction,

$$
\mathrm{AH} \longrightarrow \mathrm{A}+\mathrm{H}^{+}+\mathrm{e}^{-},
$$

is calculated as

$$
\Delta G_{\mathrm{dh}}=\mu[\mathrm{AH}]-\mu[\mathrm{A}]+\frac{1}{2} \mu\left[\mathrm{H}_{2}(\mathrm{~g})\right] .
$$

The chemical potentials $\mu$ are given by

$$
\mu=E^{\mathrm{DFT}}+\mathrm{ZPE}-T S+\Delta U^{0 \rightarrow T},
$$

where $E^{\mathrm{DFT}}$ is the total energy of the system, ZPE is the vibrational zero point energy, $T$ is the temperature, $S$ is the entropy, and $\Delta U^{0 \rightarrow T}$ is the vibrational internal energy. For adsorbed species, all degrees of freedom are assumed to be vibrational. For free species, rotational and translational entropies are also taken into account. The changes in the vibrational spectrum of the slab upon the adsorption of a molecule are neglected. ${ }^{18}$ Numerical values are given in the supplementary material.

When using the semilocal RPBE functional ${ }^{12}$ for the free energy of the OER reaction, 


$$
2 \mathrm{H}_{2} \mathrm{O}_{(\ell)} \longrightarrow \mathrm{O}_{2}(\mathrm{~g})+2 \mathrm{H}_{2}(\mathrm{~g}) \text {, }
$$

we find a value of $\Delta G_{\mathrm{OER}}=4.00 \mathrm{eV}$, considerably lower than the experimental value of $\Delta G_{\text {OER }}^{\exp }=4.92 \mathrm{eV}$. The commonly made assumption is that this is due to a limitation of the semilocal density functional in describing the $\mathrm{O}_{2}$ species. ${ }^{6}$ The conventional workaround consists in modifying the total energy of (gas phase) $\mathrm{O}_{2}$. Hence, a correction of $0.92 \mathrm{eV}$ is added to the free energy steps in Eq. (1) or (2) that involve $\mathrm{O}_{2}$. In this way, the overall reaction free energy is consistent with the experimental value of $\Delta G_{\text {OER }}$. However, it is well known that semilocal functionals, such as RPBE, fail at accurately describing the electronic structure and the charge localization in the case of semiconductors. Furthermore, the use of an ad-hoc correction for the $\mathrm{O}_{2}$ energy is unsatisfactory and it is unclear how this correction might affect the achieved results. To address these issues, we have set out to repeat all RPBE calculations in this work using hybrid functionals. The parameters in the adopted hybrid functionals have been set so as to achieve the experimental value for the OER free energy $\Delta G_{\mathrm{OER}}$. When proceeding with the same rationale as in the case of the semilocal RPBE functional, this implies that no further adhoc correction is needed to account for the $\mathrm{O}_{2}$ molecule with these hybrid functionals. While this does not signify that the energy of the $\mathrm{O}_{2}$ molecule is perfectly captured within such a scheme, it, nevertheless, provides us with the possibility of examining the robustness of our RPBE results through the use of more advanced functionals.

\section{RESULTS}

\section{A. Regular mechanism}

For each material, we first calculate the free energy steps corresponding to the regular OER mechanism in Eq. (1). When the comparison with the previous calculations is possible, our results show good agreement. ${ }^{3,19-23}$ For illustration, we show in Fig. 1 the free energy steps obtained using RPBE and hybrid functionals for a selection of the materials in the studied set. As one can see, the results achieved with the two theoretical schemes differ quite noticeable in quantitative terms. For instance, $\Delta G_{3}$ of $\mathrm{Ni}_{2} \mathrm{P}$ changes from $\sim 1.8 \mathrm{eV}$ with RPBE to $\sim 2.4 \mathrm{eV}$ with the hybrid functional. In the case of $\mathrm{IrO}_{2}$, we even observe a qualitative change with the rate limiting step shifting from $\Delta G_{4}$ with RPBE to $\Delta G_{2}$ with hybrid functionals. The
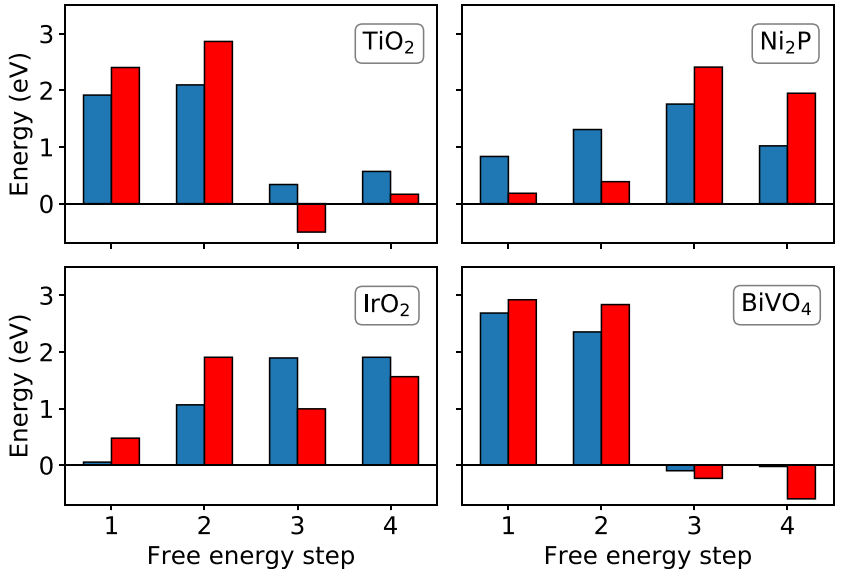

FIG. 1. Free energy steps $\Delta G_{i}, i \in\{1,2,3,4\}$ corresponding to the regular OER mechanism in Eq. (1) for four materials. Results obtained using RPBE and hybrid functionals are shown in blue and red bars, respectively.

calculated values for all materials are provided in the supplementary material.

The overpotential of the full OER is defined as $\eta \equiv \max _{i}\left\{\Delta G_{i}-1.23\right\}$ for $i \in\{1,2,3,4\}$. Figure 2 shows the calculated overpotentials as a function of the second free energy step. As found in the previous work ${ }^{3}$ and to the extent that the dominant overpotential is found in the second or third reaction step, the data can well be interpreted in terms of the linear scaling relationship, $\Delta G_{2}+\Delta G_{3}=3.2 \mathrm{eV}$. This gives calculated overpotentials showing a volcano behavior, in which the overpotential is generally dominated by $\Delta G_{3}$ for $\Delta G_{2}<1.6 \mathrm{eV}$ and by $\Delta G_{2}$ for $\Delta G_{2}>1.6 \mathrm{eV}$. According to this linear relationship, ${ }^{4}$ the lowest overpotential is found for $\Delta G_{2}=1.6 \mathrm{eV}$ and amounts to $0.37 \mathrm{eV}$.

The changes between RPBE and hybrid functional results also reflect in Fig. 2, leading to noticeable differences in both the values of $\Delta G_{2}$ and $\eta$ found for the individual materials. In particular, the order of the materials according to $\Delta G_{2}$ is not preserved. For the hybrid functional results in Fig. 2(b), all materials except $\mathrm{Ni}_{2} \mathrm{P}, \mathrm{RuO}_{2}$, and $\mathrm{BiVO}_{4}$ are limited by the second free energy step $\Delta G_{2}$ and, thus, lie on the right slope of the volcano [dotted line for $\Delta G_{2}>1.6 \mathrm{eV}$ in Fig. 2(b)]. Out of these three exceptions, only $\mathrm{Ni}_{2} \mathrm{P}$ is (a)

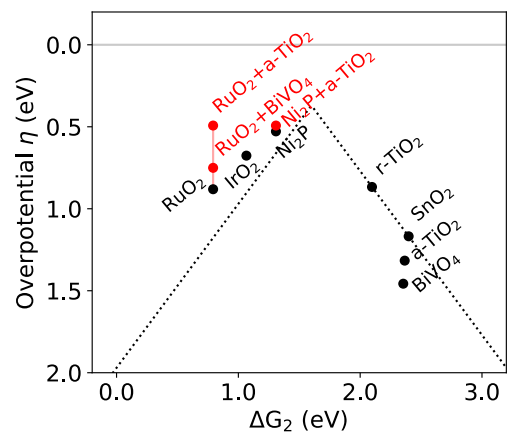

(b)

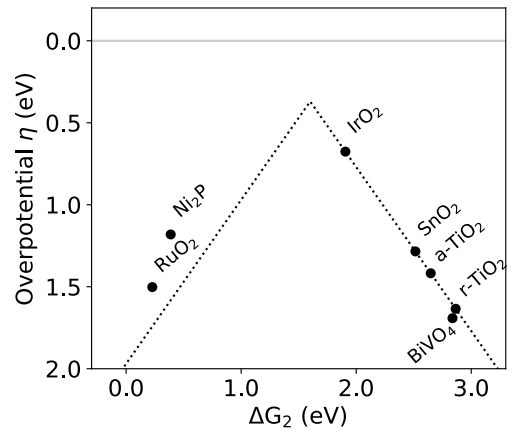

FIG. 2. Volcano plot highlighting the linear scaling relationship between the OER free energy steps corresponding to the regular mechanism. The dotted line is defined by $\max \left\{\Delta G_{2}-1.23,3.2-\Delta G_{2}-1.23\right\}$. The free energy steps are calculated with (a) the RPBE functional and (b) the hybrid functionals. The black dots correspond to the regular mechanism. The red dots correspond to the bifunctional mechanism and are only shown when this mechanism leads to a lower overpotential. 
(a)

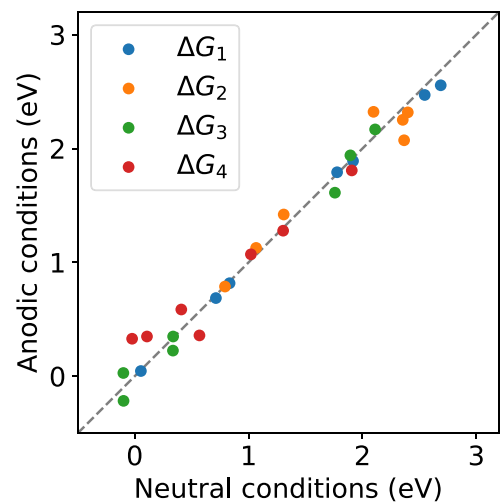

(b)

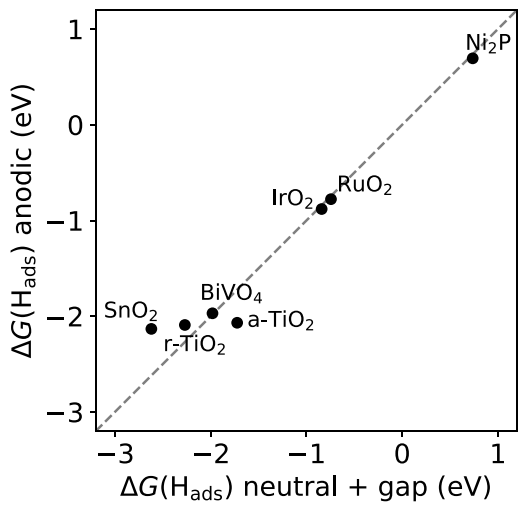

FIG. 3. (a) Comparison of the free energy steps corresponding to the regular OER mechanism obtained in neutral and anodic conditions calculated using the RPBE functional. (b) The hydrogen adsorption free energy relevant in the bifunctional mechanism as obtained under anodic conditions (vertical axis) vs the sum of the bandgap and the adsorption free energy evaluated under neutral conditions (horizontal axis). limited by $\Delta G_{3}$. Nevertheless, the calculated overpotentials for both $\mathrm{RPBE}$ and hybrid functionals are consistent with the linear scaling relationship.

The present approach of evaluating the free energy steps in neutral conditions is consistent with the previous work in the literature. ${ }^{3,19-23}$ In reality, the OER takes place under anodic conditions. To model these conditions, we remove an electron from the catalyst and perform calculations for the charged systems. Figure 3(a) draws a comparison between the results obtained for the neutral and charged simulation cells in the case of the RPBE functional showing only minor modifications. All reaction intermediates in Eq. (1) are electronegative and will attract electrons from the valence band of the bulk substrate. The binding energy of the reaction intermediates is then only slightly modified upon the introduction of a delocalized valence band hole, in agreement with our results. The small differences between the neutral and charged calculations give a mean absolute error (MAE) of $0.11 \mathrm{eV}$ and should be attributed to finite size effects associated with the finite charge density of the delocalized valence band hole.

\section{B. Bifunctional mechanism}

Next, we focus on the free energy steps corresponding to the bifunctional mechanism in Eq. (2). For every material associated with a binding site $a$, we consider the possibility that $\mathrm{H}_{\mathrm{ads}}$ in the third reaction step be adsorbed at a surface site $b$ of any other material, including itself. In all cases, an undercoordinated surface metal atom serves as site $a$ and a surface oxygen atom serves as site $b$, except in the case of $\mathrm{Ni}_{2} \mathrm{P}$, where a surface phosphorus atom is considered instead. Denoting by $N$ the number of catalysts that we investigate, this approach yields a $N \times N$ array of OER free energy steps, each element belonging to a specific combination of catalysts for the respective reactions on sites $a$ and $b$.

The bifunctional OER mechanism in Eq. (2) involves the adsorption of a hydrogen atom. The hydrogen atom, being only weakly electronegative, gives up its electron upon adsorption. In the case of a neutral system, this electron accommodates in the lowest unoccupied electronic state, which corresponds to the conduction band edge or a shallow defect state in the case of a semiconductor and to the Fermi energy in the case of a metal. Under anodic conditions, i.e., in the presence of valence band holes, the electron accommodates close to the highest occupied electronic state instead. This indicates that under anodic conditions, the hydrogen is stabilized with respect to the neutral case by an amount corresponding to the bandgap. Hence, while the regular mechanism in Eq. (1) is unaffected upon the introduction of anodic conditions, the bifunctional mechanism in Eq. (2) should be strongly affected due to the extra stabilization of the hydrogen intermediate. To verify this, we calculate
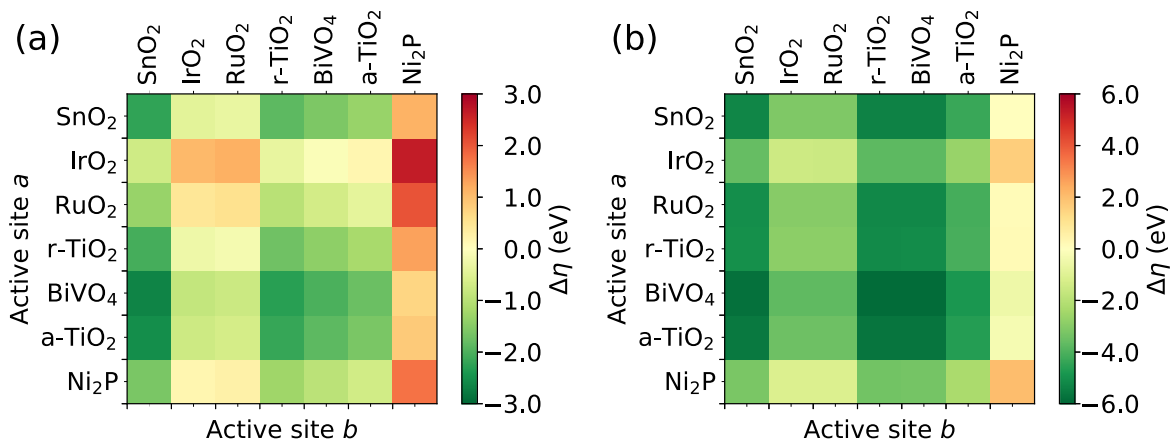

FIG. 4. The third OER free energy step $\Delta G_{3}$ for the bifunctional mechanism with respect to the regular mechanism as calculated with (a) the RPBE functional and (b) the hybrid functionals. The active sites $a$ and $b$ refer to those appearing in Eq. (2). The green boxes indicate that the corresponding combination of catalysts leads to a reduction in the third OER step in the bifunctional mechanism. 

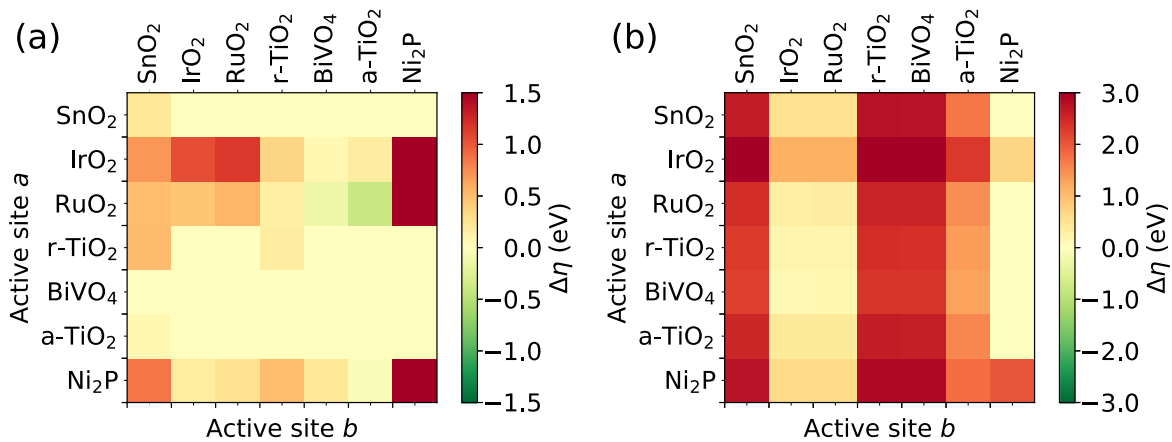

FIG. 5. The OER overpotential $\eta$ for the bifunctional mechanism with respect to the regular mechanism as calculated with (a) the RPBE functional and (b) the hybrid functionals. The green box indicates that the corresponding combination of catalysts leads to an OER overpotential reduction in the bifunctional mechanism. the hydrogen binding free energy using the RPBE functional under both neutral and anodic conditions. Figure 3(b) shows that by subtracting the bandgap from the hydrogen adsorption free energy in the case of neutral simulation cells, we obtain values close to those achieved in the presence of a valence band hole. Given the limited effect of anodic conditions on all the reaction intermediates except $\mathrm{H}_{\text {ads }}$, as evidenced by the data shown in Fig. 3(a), we henceforth retain neutral conditions for all calculations and subtract the value of the bandgap from the adsorption free energy of $\mathrm{H}_{\mathrm{ads}}$ to effectively model anodic conditions.

The differences in the third OER free energy step as calculated for the bifunctional mechanism and for the regular mechanism are shown in Fig. 4. The green boxes indicate that the bifunctional mechanism yields lower $\Delta G_{3}$ values, whereas the red boxes indicate the opposite. The grading of the intensity reflects the size of the difference. Numerical values for all cases are provided in the supplementary material. At the semilocal level of theory, we find that all materials except $\mathrm{Ni}_{2} \mathrm{P}$ lead to a reduction in $\Delta G_{3}$ when used as hydrogen acceptors (reaction at site $b$ ) in conjunction with specific other catalysts for the reaction at the site $a$. However, only when the third step is the potential limiting step, a corresponding reduction in the OER overpotential is observed, i.e., for $\mathrm{RuO}_{2}$ and $\mathrm{Ni}_{2} \mathrm{P}$. This can be seen in Fig. 5(a), where the overpotential of the full reaction for the bifunctional mechanism is compared with that of the regular reaction. Within our set of investigated catalysts, the bifunctional mechanism leads to a lower overpotential only for the particular combinations involving $\mathrm{RuO}_{2} / \mathrm{BiVO}_{4}, \mathrm{RuO}_{2} / a$ $\mathrm{TiO}_{2}$, and $\mathrm{Ni}_{2} \mathrm{P} / a-\mathrm{TiO}_{2}$, where the first material hosts site $a$ and the second one acts as a hydrogen acceptor. These favorable combinations have been added in the volcano plot of Fig. 2(a). More specifically, the pairing of $\mathrm{RuO}_{2}$ and $\mathrm{TiO}_{2}$ shows an overpotential reduction of $0.4 \mathrm{eV}$, but none of the combinations studied here leads to overpotentials above the top of the volcano in Fig. 2(a). However, our calculations suggest that such favorable pairings of materials are, indeed, possible.

In the case of hybrid functionals, we compare in Fig. 4(b) the free energy steps $\Delta G_{3}$ of the two competing mechanisms. We again find that all materials except $\mathrm{Ni}_{2} \mathrm{P}$ bind hydrogen strongly and, thus, can lead to the reduction in the third free energy step when paired with a suitable catalyst. However, except for the case of $\mathrm{Ni}_{2} \mathrm{P}$, the third free energy step $\Delta G_{3}$ does not correspond to the reaction limiting step. Hence, the reduction in this step does not affect the overpotential, as conveyed by the majority red boxes in Fig. 5(b). On the contrary, the reduction in $\Delta G_{3}$ is associated with a corresponding increase in $\Delta G_{4}$, which generally becomes the rate limiting step and, hence, determines the overpotential of the full reaction. No combination of the catalysts investigated here leads to an overpotential reduction at the hybrid functional level of theory.

Hence, this investigation demonstrates that there exist specific combinations of catalysts for which the overpotential of the OER following the bifunctional mechanism can, indeed, be reduced with respect to the regular mechanism. These combinations exhibit lower overpotentials than imposed by the linear scaling relationship and, thus, indicate that this relationship can be broken. Even though none of the cases studied here surmounts the top of the volcano, our calculations suggest that such advantageous pairings are, indeed, within reach. These are the important results that support the search for the ideal combination of materials catalyzing the OER through the bifunctional mechanism.

\section{Band edge alignment and descriptor}

In order to guide the search toward the optimal catalyst, it is important to identify relevant physical descriptors characterizing

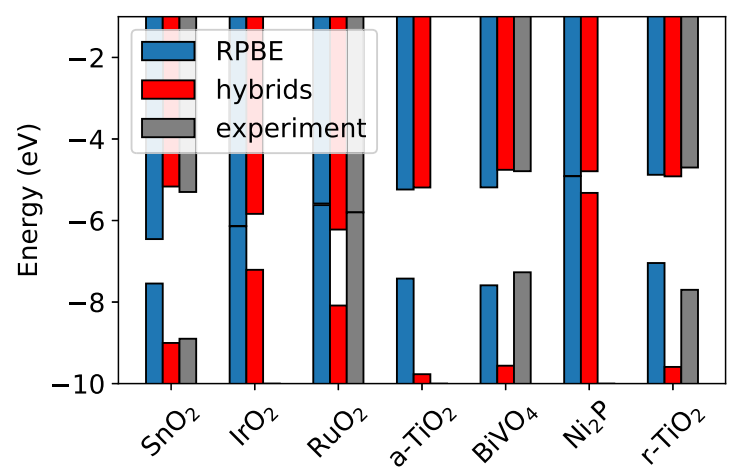

FIG. 6. Band edge alignment with respect to the vacuum level. The RPBE results are shown in blue bars, and the hybrid functional results are shown in red bars. Where available, the experimental reference is shown in gray bars. The experimental bandgap is added to the electron affinity in order to obtain the position of the valence band edge. The references are the following: $\mathrm{SnO}_{2}$, Refs. 25-27; $\mathrm{RuO}_{2}$, Ref. 28; rutile $\mathrm{TiO}_{2}$, Refs. 29 and 30; and $\mathrm{BiVO}_{4}$, Refs. 31 and 32. 
(a)

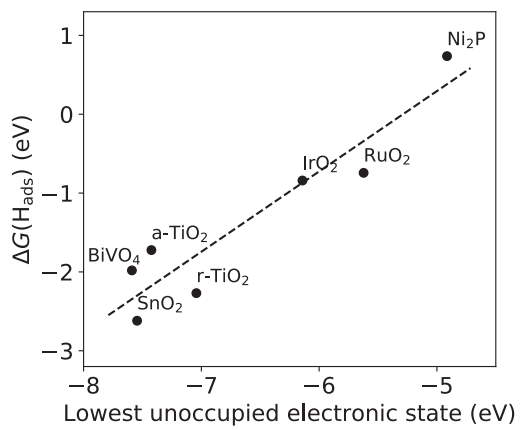

(b)

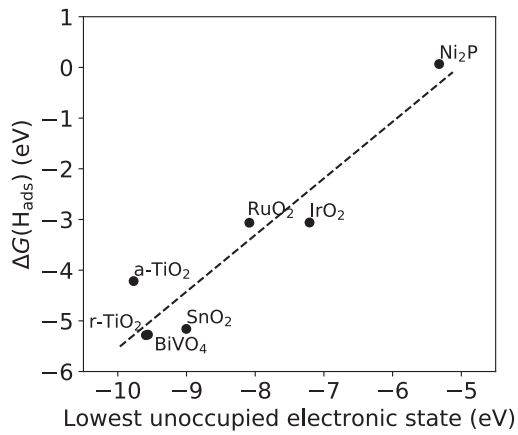

FIG. 7. Free energy of hydrogen adsorption vs highest occupied electronic level aligned with respect to the vacuum level. The dotted line represents a linear regression. The results, which correspond to anodic conditions, are achieved (a) with the RPBE functional and (b) with the hybrid functionals. the performance of a catalyst. In the bifunctional mechanism, the third OER step decreases with an increasing hydrogen adsorption free energy, which can be defined as the opposite of $\Delta G_{4}$ pertaining to the mechanism given in Eq. (2). At the same time, the hydrogen binding energy for the ideal hydrogen acceptor should not be too large since otherwise the fourth step would turn unfavorable. For instance, $\mathrm{SnO}_{2}, \mathrm{TiO}_{2}$, and $\mathrm{BiVO}_{4}$ are examples of materials with strong hydrogen adsorption free energy leading to large reductions in $\Delta G_{3}$ when used as hydrogen acceptors. The hydrogen binding energy could, thus, be used as a descriptor of the suitability of a material as a hydrogen acceptor within the bifunctional scheme.

The case $\mathrm{SnO}_{2}$, which is characterized by a deep position of the valence band edge when the material is exposed to an aqueous solution, suggests that the hydrogen adsorption free energy can be associated with the energy level of the highest occupied electronic state. Indeed, a similar correlation between hydrogen adsorption and the lowest unoccupied electronic state has been studied in the case of oxidative dehydrogenation catalyzed by vanadia and ceria. ${ }^{24}$

To verify this connection, we calculate for all materials in the studied set the alignment of the band edges with respect to the vacuum level. We proceed as follows. First, we establish the energy level of the highest occupied electronic state with respect to the average electrostatic potential by modeling the bulk material in a periodic simulation cell. Next, we consider a slab of the material in vacuum and calculate the line-up of the average electrostatic potential between the bulk-like central region of the slab and the vacuum region. By combining these results, we can position the highest occupied electronic level with respect to the vacuum level. This level corresponds to the valence band edge in the case of semiconductors (ionization potential) and to the Fermi level in the case of metals (work function). All these calculations are carried out in neutral charge conditions.

The calculated values obtained with RPBE and hybrid functionals are shown in Fig. 6 in comparison with available experimental values. We find that the hybrid functionals lead to a better agreement with the experiment for the lowest unoccupied electronic level, except for $\mathrm{RuO}_{2}$. The data shown in Fig. 6 result in a mean absolute error (MAE) of $0.49 \mathrm{eV}$ in the case of RPBE, as compared to $0.27 \mathrm{eV}$ in the case of hybrid functionals. Both functionals perform poorly when it comes to determine the position of the highest occupied electronic level. The RPBE functional gives a MAE of $0.63 \mathrm{eV}$ due to the systematic tendency of placing this level too high. The hybrid functionals perform even worse pushing the highest occupied level well below the experimental value, with a corresponding MAE of $1.64 \mathrm{eV}$. This shortcoming of the hybrid functionals should be attributed to the rather high fractions of Fock exchange $\alpha$ used here. Indeed, for most of the studied materials, the fraction $\alpha$ that reproduces the experimental bandgap is lower than the one adopted here. The overestimation of $\alpha$ leads to an excessive opening of the bandgap and consequently to a deeper energy level for the highest occupied electronic state. The numerical values shown in Fig. 6 are provided in the supplementary material.

Figure 7 shows a clear correlation between the hydrogen adsorption free energy and the highest occupied electronic level aligned with respect to the vacuum level: the lower the energy level, the stronger the hydrogen binding energy. The correlation holds for both levels of theory considered here despite their inherent errors. The energy level of the highest occupied electronic state can, thus, be used as a valid descriptor of the potential of a material for acting as a hydrogen acceptor within the framework of the bifunctional mechanism. More specifically, because of its association with $\Delta G_{4}$, a suitable hydrogen binding free energy in the bifunctional OER pathway should not be much higher than $1.23 \mathrm{eV}$ to avoid disfavoring the last OER step. The correlation in Fig. 7 indicates that the ideal hydrogen acceptor should exhibit a valence band edge lying at $\sim 6.5 \mathrm{eV}$ below the vacuum level. This condition suggests that typical oxide insulators such as $\mathrm{SnO}_{2}, \mathrm{BiVO}_{4}$, and $\mathrm{TiO}_{2}$ generally bind hydrogen too strongly, while $\mathrm{Ni}_{2} \mathrm{P}$ shows the opposite behavior. In our set of materials, the middle region around $6.5 \mathrm{eV}$ is occupied by the metals $\mathrm{IrO}_{2}$ and $\mathrm{RuO}_{2}$, albeit the two adopted levels of theory show noticeable variation in the results. In this respect, one should remind that the use of hybrid functionals results in the opening of a bandgap for these metals and might, thus, be less reliable than RPBE in these cases.

\section{DISCUSSION AND CONCLUSION}

Both the semilocal and hybrid-functional results suggest that the bifunctional mechanism could, indeed, lead to a reduction in the OER overpotential with respect to the regular mechanism. Our study allows us to establish that the bifunctional mechanism is more favorable when the following criteria are met. First, the potential determining step in the regular mechanism must correspond to the 
third one, i.e., the formation of the $\mathrm{OOH}_{\mathrm{ads}}$ intermediate. Second, the hydrogen acceptor should bind the hydrogen strongly, resulting in a sizable reduction in the third step in Eq. (2) as compared to the third step in Eq. (1). Third, the magnitude of the reduction should at the same time not be excessive to prevent the fourth step in Eq. (2) from becoming the reaction limiting one. Since the reduction in the overpotential is generally limited by the linear scaling relationship involving the second and third steps, the fulfillment of these criteria opens the way to a competitive bifunctional mechanism, as proposed by Song et al.

To be more specific, the correlation between the hydrogen adsorption free energy and the position of the highest occupied electronic level suggests that the ideal hydrogen acceptor (representing site $b$ in the bifunctional mechanism) exhibits a valence band edge at around $\sim 6.5 \mathrm{eV}$ below the vacuum level. In this way, the hydrogen adsorption free energy, which corresponds to $-\Delta G_{4}$ in the bifunctional scheme, achieves a value close to the optimal $1.23 \mathrm{eV}$ and does not lead to any extra overpotential. In regard to the optimal catalyst providing the active site $a$, the first and second reaction steps should exhibit free energy steps close to $1.23 \mathrm{eV}$ with the rate limiting step being the third one. In such a scenario, the bifunctional scheme could ideally lead to an arbitrarily low OER overpotential.

Our calculations show a strong influence of the adopted exchange and correlation functional. We evaluate all energies using both the semilocal RPBE functional and modified hybrid functionals in which the fraction of exact exchange is chosen in order to recover the overall OER free energy. The use of hybrid functionals allows us to avoid ad-hoc corrections, which are necessary at the semilocal level of theory. However, this leads to fractions of Fock exchange $\alpha$ that result in severe overestimations of the bandgap for several materials in the set under consideration. By consequence, the hybrid functionals generally result in overvalued binding free energies of hydrogen. This point of contention can presently not be settled with available functionals. Nevertheless, the comparison between semilocal and hybrid functional free energies allows one to infer the degree of uncertainty in the theoretical predictions.

We remark that the detailed reaction mechanism has not been addressed in this work. It, therefore, remains an open question to what extent the two active sites $a$ and $b$ can be brought in proximity in order to promote the reaction through the bifunctional mechanism. The charge states of the active sites and the electronic configuration of the catalyst can have a considerable effect on the catalytic activity. The detailed free energy steps can be very sensitive to the particular atomic configuration. For instance, the size of the nanoparticles, their stoichiometry, the way they are bound to the substrate, the presence of spectator defects, the surface modifications of the substrate, and the proximity of the two active sites are all critical aspects to be considered when building a suitable combination of catalysts for the bifunctional mechanism. In the absence of any detailed structural description of an experimental realization, it is presently untimely to address the reaction pathway in a realistic modeling approach. In particular, all such structural details are known to significantly affect the binding energies of the OER intermediates and, consequently, the associated free energy in the regular mechanism. Nevertheless, general properties such as the linear scaling laws remain largely unperturbed overall. ${ }^{3,33}$ In the same way, we expect that the insight provided by our study of model surfaces is of value when searching for suitable catalysts for the bifunctional mechanism.

Along the same line, realistic catalyst surfaces may also present a variety of surface polarons affecting the calculated free energy steps. In particular, several theoretical investigations have highlighted the role of such polarons in water-splitting processes. ${ }^{34-39}$ In the specific case of rutile $\mathrm{TiO}_{2}$, hole polarons have been found to play a critical role in initiating the water splitting reaction ${ }^{40}$ and in modifying the OER free energy steps. ${ }^{35,41}$ The stability of polarons and their effect on the OER activity are material specific and should be considered in future investigations of detailed reaction pathways.

Likewise, our present work does not include the explicit treatment of the solvent. The effect of the solvent on the OER free energies has been investigated in detail in the specific case of rutile $\mathrm{TiO}_{2}{ }^{42,43}$ Cheng et al. have considered neutral OER intermediates and have found the effect of the solvent on the OER free energies to be limited. ${ }^{42}$ Subsequent studies have shown that charging effects due to the solvent affect the second and third OER steps by up to $0.5 \mathrm{eV}$ but do not invalidate the linear scaling relationship. ${ }^{43}$ Similarly, the consideration of solvation effects in the case of $\mathrm{IrO}_{2}$ results in a marginal effect on the OER overpotential. ${ }^{23}$ Therefore, the linear scaling relationships are expected to hold even when the solvent is explicitly considered. Regarding the effect of the solvent on the binding energy of $\mathrm{H}_{\mathrm{ads}}$, which assumes a critical role within the bifunctional mechanism, comprehensive calculations on platinum suggest a solvent effect on the order of $\sim 0.1 \mathrm{eV} .{ }^{44}$ Based on these considerations, we expect the general conclusions drawn from the trends observed in this work to remain valid.

To sum up, we studied the energetic viability of the bifunctional mechanism for the oxygen evolution reaction for a variety of catalyst materials, at both the semilocal and hybrid functional levels of theory. We found that the bifunctional mechanism may lead to the reduction in the OER overpotential for specific combinations of catalysts. At the same time, we observed that the particular functional used is critical for the identification of such favorable pairings of catalysts. Irrespective of the adopted functional, we, nevertheless, highlight a correlation between the energy level of the highest occupied electronic state and the hydrogen adsorption free energy. In this way, the present work provides a descriptor to guide the search for suitable hydrogen acceptors within the framework of this mechanism. Overall, our work provides support to the bifunctional mechanism as a means to break the linear scaling relationships, which otherwise hinder further reductions in the OER overpotential.

\section{SUPPLEMENTARY MATERIAL}

The supplementary material contains calculated values for the thermodynamic corrections, for the free energy steps in the regular and the bifunctional mechanisms, and for the bandgap and the band edges.

\section{ACKNOWLEDGMENTS}

The authors thank M. Busch and C. Corminboeuf for useful interactions. This work has been realized in relation to the National 
Center of Competence in Research (NCCR) "Materials': Computational Design and Discovery of Novel Materials (MARVEL)" of the SNSF. The authors used computational resources of the Swiss National Supercomputing Centre (CSCS Grant under Project Nos. s879 and mr24) and of the Scientific IT and Application Support Center (SCITAS) of the Ecole Polytechnique Fédérale de Lausanne.

\section{REFERENCES}

${ }^{1}$ P. Lianos, Appl. Catal., B 210, 235 (2017).

${ }^{2}$ J. H. Montoya, L. C. Seitz, P. Chakthranont, A. Vojvodic, T. F. Jaramillo, and J. K. Nørskov, Nature 16, 70 (2017).

${ }^{3}$ I. C. Man, H.-Y. Su, F. Calle-Vallejo, H. A. Hansen, J. I. Martínez, N. G. Inoglu, J. Kitchin, T. F. Jaramillo, J. K. Nørskov, and J. Rossmeisl, ChemCatChem 3, 1159 (2011).

${ }^{4}$ J. K. Nørskov, T. Bligaard, A. Logadottir, S. Bahn, L. B. Hansen, M. Bollinger, H. Bengaard, B. Hammer, Z. Sljivancanin, M. Mavrikakis, Y. Xu, S. Dahl, and C. J. H. Jacobsen, J. Catal. 209, 275 (2002).

${ }^{5}$ H. Oberhofer, in Handbook of Materials Modeling: Applications: Current and Emerging Materials, edited by W. Andreoni and S. Yip (Springer International Publishing, Cham, 2018), pp. 1-33.

${ }^{6}$ J. K. Nørskov, J. Rossmeisl, A. Logadottir, L. Lindqvist, J. R. Kitchin, T. Bligaard, and H. Jónsson, J. Phys. Chem. B 108, 17886 (2004).

${ }^{7}$ R. Christensen, H. A. Hansen, C. F. Dickens, J. K. Nørskov, and T. Vegge, J. Phys. Chem. C 120, 24910 (2016).

${ }^{8}$ F. Song, M. M. Busch, B. Lassalle-Kaiser, C.-S. Hsu, E. Petkucheva, M. Bensimon, H. M. Chen, C. Corminboeuf, and X. Hu, ACS Cent. Sci. 5, 558 (2019).

${ }^{9}$ Z. Guo, F. Ambrosio, W. Chen, P. Gono, and A. Pasquarello, Chem. Mater. 30, 94 (2018).

${ }^{10}$ Y.-H. Li, X. G. Gong, and S.-H. Wei, Phys. Rev. B 73, 245206 (2006).

${ }^{11}$ J. Hutter, M. Iannuzzi, F. Schiffmann, and J. VandeVondele, Science 4, 15 (2014).

${ }^{12}$ B. Hammer, L. B. Hansen, and J. K. Nørskov, Phys. Rev. B 59, 7413 (1999).

${ }^{13}$ S. Goedecker, M. Teter, and J. Hutter, Phys. Rev. B 54, 1703 (1996).

${ }^{14}$ J. P. Perdew, M. Ernzerhof, and K. Burke, J. Chem. Phys. 105, 9982 (1996).

${ }^{15}$ A. V. Krukau, O. A. Vydrov, A. F. Izmaylov, and G. E. Scuseria, J. Chem. Phys. 125, 224106 (2006).

${ }^{16}$ J. Heyd, G. E. Scuseria, and M. Ernzerhof, J. Chem. Phys. 118, 8207 (2003).

${ }^{17}$ M. Guidon, J. Hutter, and J. VandeVondele, J. Chem. Theory Comput. 6, 2348 (2010).
${ }^{18}$ A. A. Peterson, F. Abild-Pedersen, F. Studt, J. Rossmeisl, and J. K. Nørskov, Energy Environ. Sci. 3, 1311 (2010).

${ }^{19}$ J. Rossmeisl, Z. W. Qu, H. Zhu, G. J. Kroes, and J. K. Nørskov, J. Electroanal. Chem. 607, 83 (2007).

${ }^{20}$ Y.-F. Li, Z.-P. Liu, L. Liu, and W. Gao, J. Am. Chem. Soc. 132, 13008 (2010).

${ }^{21}$ J. Hu, X. Zhao, W. Chen, H. Su, and Z. Chen, J. Phys. Chem. C 121, 18702 (2017).

${ }^{22}$ Á. Valdés, Z.-W. Qu, G.-J. Kroes, J. Rossmeisl, and J. K. Nørskov, J. Phys. Chem. 112, 9872 (2008).

${ }^{23}$ J. A. Gauthier, C. F. Dickens, L. D. Chen, A. D. Doyle, and J. K. Nørskov, J. Phys. Chem. 121, 11455 (2017).

${ }^{24}$ X.-P. Wu and X.-Q. Gong, J. Am. Chem. Soc. 137, 13228 (2015).

${ }^{25}$ A. Klein, C. Körber, A. Wachau, F. Säuberlich, Y. Gassenbauer, R. Schafranek, S. P. Harvey, and T. O. Mason, Thin Solid Films 518, 1197 (2009).

${ }^{26}$ Z. M. Jarzebski and J. P. Morton, J. Electrochem. Soc. 123, 333C (1976).

${ }^{27}$ K. Reimann and M. Steube, Solid State Commun. 105, 649 (1998).

${ }^{28}$ A. Böttcher and H. Niehus, Phys. Rev. B 60, 14396 (1999).

${ }^{29}$ K. D. Schierbaum, S. Fischer, M. C. Torquemada, J. L. de Segovia, E. Román, and J. A. Martín-Gago, Surf. Sci. 345, 261 (1996).

${ }^{30}$ H. Tang, K. Prasad, R. Sanjinès, P. E. Schmid, and F. Lévy, J. Appl. Phys. 75, 2042 (1994).

${ }^{31}$ R. Crespo-Otero and A. Walsh, J. Phys. Chem. Lett. 6, 2379 (2015).

${ }^{32}$ J. K. Cooper, S. Gul, F. M. Toma, L. Chen, P.-A. Glans, J. Guo, J. W. Ager, J. Yano, and I. D. Sharp, Chem. Mater. 26, 5365 (2014).

${ }^{33}$ H.-Y. Su, Y. Gorlin, I. C. Man, F. Calle-Vallejo, J. K. Nørskov, T. F. Jaramillo, and J. Rossmeisl, Phys. Chem. Chem. Phys. 14, 14010 (2012).

${ }^{34}$ D. Wang, H. Wang, and P. Hu, Chem. Phys. 17, 1549 (2015).

${ }^{35}$ J. Cheng, J. VandeVondele, and M. Sprik, J. Phys. Chem. 118, 5437 (2014).

${ }^{36} \mathrm{C}$. Di Valentin and A. Selloni, Chem. Lett. 2, 2223 (2011).

${ }^{37}$ J. Wiktor, F. Ambrosio, and A. Pasquarello, ACS Energy Lett. 3, 1693 (2018).

${ }^{38} \mathrm{~J}$. Wiktor and A. Pasquarello, ACS Appl. Mater. Interfaces 11, 18423 (2019).

${ }^{39}$ F. Ambrosio and J. Wiktor, J. Phys. Chem. Lett. 10, 7113 (2019).

${ }^{40}$ J. Cheng, M. Sulpizi, J. VandeVondele, and M. Sprik, ChemCatChem 4, 636 (2012).

${ }^{41}$ P. Gono, J. Wiktor, F. Ambrosio, and A. Pasquarello, ACS Catal. 8, 5847 (2018).

${ }^{42}$ J. Cheng, X. Liu, J. A. Kattirtzi, J. VandeVondele, and M. Sprik, Angew. Chem., Int. Ed. 53, 12046 (2014).

${ }^{43}$ P. Gono, F. Ambrosio, and A. Pasquarello, J. Phys. Chem. C 123, 18467 (2019).

${ }^{44}$ I. Hamada and Y. Morikawa, J. Phys. Chem. C 112, 10889 (2008). 